\title{
High-selectivity neural probe based on a Fabry-Perot optical filter and a CMOS silicon photodiodes array at visible wavelengths
}

Sara Pimenta

José P. Pereira

Nuno M. Gomes

João F. Ribeiro

Manuel F. Silva

Sandra B. Goncalves

Graca Minas

José H. Correia 


\title{
High-selectivity neural probe based on a Fabry-Perot optical filter and a CMOS silicon photodiodes array at visible wavelengths
}

\author{
Sara Pimenta, ${ }^{*}$ José P. Pereira, Nuno M. Gomes, João F. Ribeiro, Manuel F. Silva, Sandra B. Goncalves, \\ Graca Minas, and José H. Correia \\ University of Minho, CMEMS-UMinho, Campus de Azurém, Guimarães, Portugal
}

\begin{abstract}
This paper presents a silicon neural probe with a high-selectivity optical readout function and light emitting diodes for neurons photostimulation and fluorophore excitation. A high-selectivity Fabry-Perot optical filter on the top of a CMOS silicon photodiodes array can read the emitted fluorescence, which indicates the neurons physiological state. The design, fabrication, and characterization of the optical filter are presented. The $\mathrm{SiO}_{2} / \mathrm{TiO}_{2}$ based optical filter thin films were deposited by RF sputtering. The performance of the optical filter deposited on the top of the silicon photodiodes array, implemented in the neural probe, was tested through in-vitro fluorescence measurements. The transmittance peak of the fabricated optical filter is $81.8 \%$ at $561 \mathrm{~nm}$, with a full width at half maximum of $28 \mathrm{~nm}$. The peak responsivity of the CMOS silicon photodiode with the optical filter deposited on its top is $273.6 \mathrm{~mA} / \mathrm{W}$ at $578 \mathrm{~nm}$. The in-vitro fluorescence measurements results show a CMOS photodiode current proportional to the fluorophore concentration with a good linearity $\left(R^{2}=0.9361\right)$. The results validate the use of the neural probe with the high-selectivity optical readout function to determine the presence of different fluorophore concentrations. The development of the device in a conventional CMOS process allows on-chip electronics readout. @ 2018 Society of Photo-Optical Instrumentation Engineers (SPIE) [DOI: 10.1117/1.JBO.23.10.105004]

Keywords: high-selectivity neural probe; CMOS silicon photodiodes array; Fabry-Perot optical filter; in-vitro fluorescence measurements.
\end{abstract}

Paper 180270RR received May 8, 2018; accepted for publication Sep. 25, 2018; published online Oct. 22, 2018.

\section{Introduction}

Globally, millions of people suffer from mental disorders, such as schizophrenia, affecting about 23 million people; dementia, affecting 50 million people; bipolar affective disorder, which affects about 60 million people; and depression, which affects 300 million people. ${ }^{1}$

In basic neuroscience, implantable neural electrodes are broadly used to study the function and behavior of the central nervous system. ${ }^{2}$ Brain-computer interfaces (such as neural probes) interact with the central nervous system to simplify understanding, mitigating, and treating neurological disorders. ${ }^{3}$

New techniques have emerged from the scientific development of neuroscience, such as optogenetics. Optogenetics has become a widely used technique for the study of the brain dynamics and circuitry of neurological disorders. ${ }^{4}$ In optogenetics, neuron cells are genetically modified to make them light sensitive, providing excitatory or inhibitory control of the neural activity with high spatiotemporal specificity. ${ }^{5,6}$ Accordingly, opsins are introduced into the brain. ${ }^{5}$ The targeted neuron cells express the introduced opsin and, when exposed to light in a specific wavelength, a biological effect on those specific cells is promoted. ${ }^{5}$ Thus, the rise of optogenetics has opened the chance to use light to address and generate action potentials in specific cells. ${ }^{7}$ For example, the channelrhodopsin-2 (ChR2) is a protein whose cation channel is directly activated by blue light (at $\sim 470 \mathrm{~nm}$ ). ${ }^{8} \mathrm{ChR} 2$ allows the cation inflow of $\mathrm{Ca}^{2+}$, promoting cell depolarization. ${ }^{9}$ In addition to

*Address all correspondence to: Sara Pimenta, E-mail: sara.pimenta@ dei uminho.pt
$\mathrm{Ca}^{2+}$ inflow, after the blue light stimulation, it is possible to use a fluorophore coelectroporated into the neuron cells, such as a genetically encoded calcium indicator for optical imaging (GECO). ${ }^{9}$ The O-GECO1 is such an example, it is a fluorophore with an increased capacity of fluorescence when bonded to $\mathrm{Ca}^{2+} \cdot{ }^{10}$ When O-GECO1 is bonded to $\mathrm{Ca}^{2+}$ and it is excited with green light (at $\sim 540 \mathrm{~nm}$ ), it emits fluorescence with an emission peak at $\sim 570 \mathrm{~nm} .{ }^{10,11}$ As a result, neuron cells respond to photostimulation and their physiological state can be determined based on fluorescence. ${ }^{9}$

Several authors have developed neural devices with CMOS image sensors for fluorescence imaging or intrinsic signal imaging. ${ }^{12,13}$ Kobayashi et al. ${ }^{9}$ reported an implantable device with a CMOS image sensor and dual light emitting diodes (LEDs) for photostimulation and fluorescence imaging. The authors reported the use of blue LEDs for photostimulation, green LEDs for fluorescence excitation, and an absorption filter (long-pass liquid photoresist filter: $>600 \mathrm{~nm}$ ) superimposed on the CMOS image sensor, to pass the fluorescence and intercept the stimulation and excitation light. The bottleneck of these solutions is the selectivity of the optical readout function for different wavelengths in the visible region of the spectrum.

Thus, in this paper it is presented the fabrication of a silicon neural probe (see Fig. 1) with LEDs for photostimulation and fluorophore excitation. A CMOS silicon photodiodes array was also implemented in the neural probe to detect the emitted fluorescence and a high-selectivity Fabry-Perot optical filter was deposited on top of the photodiodes array. The extracted

$1083-3668 / 2018 / \$ 25.00$ @ 2018 SPIE 


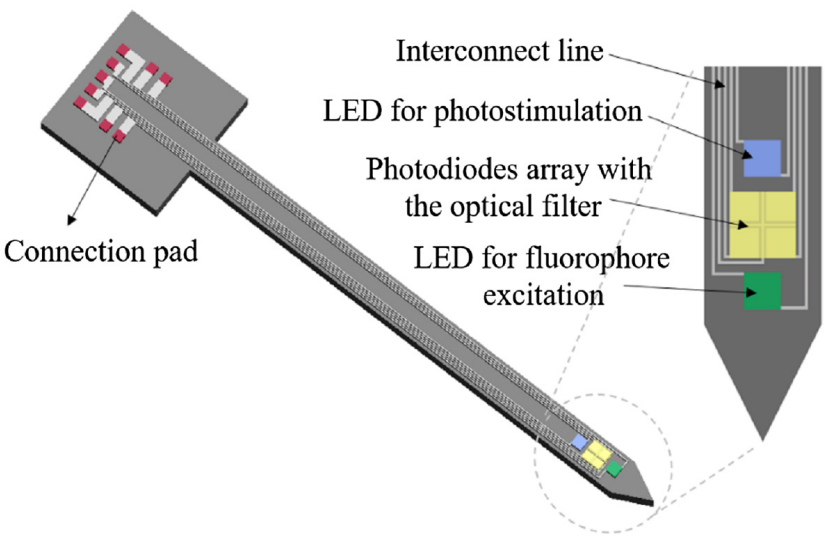

Fig. 1 Design of the optical silicon neural probe with: LEDs and the CMOS silicon photodiodes array with the high-selectivity Fabry-Perot optical filter deposited on its top.

fluorescence signal will be more reliable, selective, and sensitive to small intensity emissions if the optical filter takes into account the emission peak of the molecule, whose emitted fluorescence will be detected $(\sim 570 \mathrm{~nm}$ for the O-GECO1). This paper presents the design, fabrication, and characterization of an optical filter for the reported application. Moreover, the validation of the fabricated optical filter on the top of a CMOS silicon photodiodes array implemented in the neural probe was achieved, performing in-vitro fluorescence measurements with a fluorophore with excitation and emission properties similar to the O-GECO1. This is the first description of a silicon neural probe with a high-selectivity optical readout function.

\section{Design and Fabrication}

\subsection{CMOS Silicon Photodiodes Array}

The CMOS silicon photodiodes array $(2 \times 2)$ was designed and fabricated in a $p$-type silicon wafer. The photodiodes are $n+/ p$-epilayer type, which are the ones more suitable for the visible spectral region. Each photodiode active area is $100 \mu \mathrm{m} \times 100 \mu \mathrm{m}$. The measured responsivity as a function of the wavelength is shown in Fig. 2. The responsivity measurement was performed using a pinhole with $100-\mu \mathrm{m}$ diameter in one commercial photodiode (Hamamatsu S1336-5BQ), used as a reference to extract the CMOS photodiode responsivity curve. Moreover, the measurement was performed using an optical setup comprising: a quartz tungsten halogen lamp at $200 \mathrm{~W}$ (Newport 6334NS) with a light incident angle of $0 \mathrm{deg}$, a picoammeter (Keithley 487), a monochromator (Newport 74125), and an optical fiber (Newport 77563). The responsivity experimental measurement was performed with 0-deg light incident angle, which is appropriated to the final device configuration, where the illumination is in the same face of the detection.

The CMOS silicon photodiodes array was fabricated in a conventional double-metal single-polysilicon $0.7-\mu \mathrm{m} n$-well CMOS process. After completion of the standard CMOS process, a postprocessing module for deposition of thin films and blade dicing techniques were performed to produce a neural probe with the pad contacts for the commercial GaN/sapphire LEDs. The neural probe pads, the interconnected lines, and the contacts were made of titanium (15-nm thick), aluminum (200-nm thick), and platinum (60-nm thick) thin films, patterned with photolithography techniques and deposited with e-beam

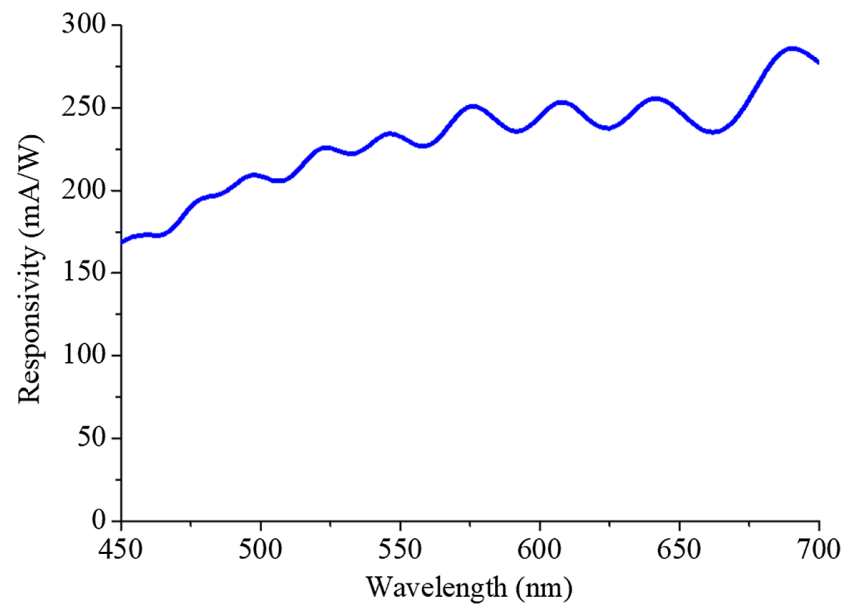

Fig. 2 Responsivity as a function of wavelength for the CMOS silicon photodiode.

(titanium and aluminum) and DC sputtering (platinum). Blade dicing allowed to define the probe geometry. All cuts were carried out by a high-precision DAD 2H/6T (DISCO Corporation) dicing saw, with a blade (NBC-ZB 2050, DISCO) suitable for silicon samples sawing. ${ }^{14}$ Cutting parameters were: (1) $0.3 \mathrm{~mm} \cdot \mathrm{s}^{-1}$ speed; (2) $Z$-axis step $=0 \mathrm{~mm}$, which determines the maximum cut depth of the blade as the neural probe is expected to be detached from the wafer; (3) $X$-axis step $=50 \mathrm{~mm}$, it sets the length of the cut, and therefore the length of the probe $(8 \mathrm{~mm})$; and (4) $Y$-axis step $=$ $700 \mu \mathrm{m}$, which establishes the probe width.

\subsection{Optical Filter Structure and Simulation}

The Fabry-Perot structure is a simple interferometer, where the incident light suffers multiple reflections between the coated surfaces that define the cavity. The emerging wavefronts interfere constructively only if there is no phase difference between them. At other wavelengths, destructive interference of the transmitted wavefronts reduces the transmitted intensity toward to zero. Therefore, this device acts as a filter that transmits certain wavelengths and reflects the others back to the light source.

The implemented Fabry-Perot optical filter has dielectric mirrors, featuring low-energy absorption rates, and high transmittance at a specific range of wavelengths. ${ }^{15}$ The optical filter multilayer is composed of 11 thin films with high and low refractive index materials, alternatively (see Fig. 3).

The Fabry-Perot operation principle is presented in Eq. (1):

$\lambda q=2 n_{r} d_{r}$

where $\lambda$ is the transmitted wavelength, $q$ is the filter interference order (it was used $q=1$ ), $n_{r}$ is the refractive index of the resonant cavity material, and $d_{r}$ is the thickness of the resonant cavity. ${ }^{15}$

The thickness of each film $(d)$ that forms each mirror multilayer is also related to the transmitted wavelength $(\lambda)$, considering Eq. (2):

$\lambda=4 n d$, 


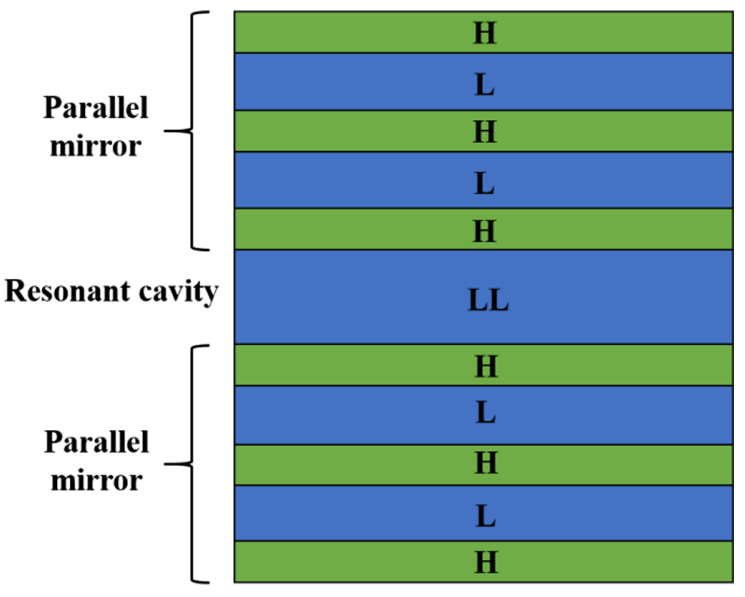

Fig. 3 Structure of the optical filter with dielectric mirrors: $\mathrm{H}-$ mirror layer with high refractive index material, L-mirror layer with low refractive index material, and $\mathrm{LL}$-resonant layer with low refractive index material.

For the reported application, it is expected to have an optical filter with a transmittance peak close to $570 \mathrm{~nm}$. The optical filter was computationally designed using the software TFCalc $^{\mathrm{TM}} 3.5$, in which the experimental refractive indices of the used materials were set in the database. The dielectric materials used as high and low refractive index materials were $\mathrm{TiO}_{2}$ and $\mathrm{SiO}_{2}$, respectively. The experimental refractive indices of these materials are reported in Ref. 16 (1.47 for $\mathrm{SiO}_{2}$ and 2.4 for $\mathrm{TiO}_{2}$, at $550 \mathrm{~nm}$ ), considering the fabrication process of the optical filter, RF sputtering (see Sec. 2.3).

The resonant cavity thickness and the thicknesses of each thin film that forms each mirror multilayer are shown in Table 1. The thicknesses of each film that forms each mirror multilayer were calculated using as reference a central wavelength of $550 \mathrm{~nm}$ and Eq. (2). The resonant cavity thickness was obtained using Eq. (1) and adjusted on TFCalc ${ }^{\mathrm{TM}} 3.5$ software.

Table 1 Optical filter layers thickness.

\begin{tabular}{lc} 
Material & Thickness (nm) \\
\hline $\mathrm{TiO}_{2}$ & 57 \\
$\mathrm{SiO}_{2}$ & 94 \\
$\mathrm{TiO}_{2}$ & 57 \\
$\mathrm{SiO}_{2}$ & 94 \\
$\mathrm{TiO}_{2}$ & 57 \\
$\mathrm{SiO}_{2}$ & 206 \\
$\mathrm{TiO}_{2}$ & 57 \\
$\mathrm{SiO}_{2}$ & 94 \\
$\mathrm{TiO}_{2}$ & 57 \\
$\mathrm{SiO}_{2}$ & 94 \\
$\mathrm{TiO}_{2}$ & 57 \\
\hline
\end{tabular}

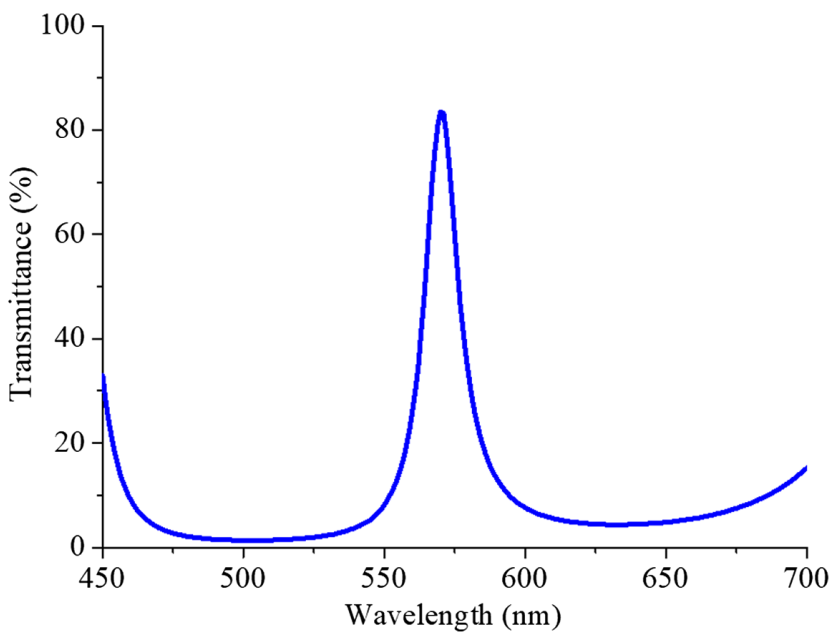

Fig. 4 Transmittance of the simulated optical filter extracted from TFCalc ${ }^{\mathrm{TM}} 3.5$.

The simulation result (from TFCalc ${ }^{\mathrm{TM}} 3.5$ ) is shown in Fig. 4. The transmittance of the optical filter at $570 \mathrm{~nm}$ is $\sim 83.5 \%$, with a full width at half maximum (FWHM) of $\sim 15 \mathrm{~nm}$.

\subsection{Optical Filter Fabrication}

Both $\mathrm{SiO}_{2}$ and $\mathrm{TiO}_{2}$ thin films were deposited by RF sputtering. The $\mathrm{SiO}_{2}$ thin films were deposited from a ceramic target $\left(\mathrm{SiO}_{2}\right.$, 99.995\% pure) with $150 \mathrm{~W}$ power supply. An Ar flow rate of $15 \mathrm{sccm}$ was used and a pressure of $8.5 \times 10^{-4}$ mbar was maintained during deposition. A deposition rate of $0.6 \AA / \mathrm{s}$ was measured through a quartz crystal sensor. The $\mathrm{TiO}_{2}$ thin films were deposited from a metallic target (Ti, 99.7\% pure) with $200 \mathrm{~W}$ power supply. The reactive RF sputtering was used with $\mathrm{Ar}+$ $\mathrm{O}_{2}$ flow rate atmosphere of 10 and $2 \mathrm{sccm}$, respectively. A pressure of $2 \times 10^{-3}$ mbar was maintained during deposition and a deposition rate of $0.15 \AA / s$ was measured through a quartz crystal sensor.

The optical filter was deposited on the neural probe (on the top of the CMOS silicon photodiodes array) and in a borosilicate glass substrate (for further optical transmittance characterization). The interface between the optical filter and the CMOS silicon photodiodes is a very thin (to reduce its optical influence on the spectral response) oxide passivation layer, done as a final step of the CMOS process.

\section{Optical Filter Characterization: Results and Discussion}

\subsection{Optical Filter Thin Films}

The thickness of the deposited $\mathrm{TiO}_{2}$ and $\mathrm{SiO}_{2}$ thin films were measured using a profilometer (Veeco Dektak 150). Table 2 shows the experimental thickness obtained for each layer. Comparing the obtained results with the theoretical thicknesses shown in Table 1, there are some differences that can be related with the calibration of the quartz crystal sensor used for real-time thickness measurement during each deposition. Additionally, a slight reduction in the resonant cavity thickness was performed intentionally during the depositions, considering an increase in the thickness of each film that forms the first mirror multilayer and its affectation on the final optical filter 
Table 2 Experimental optical filter layers thickness.

\begin{tabular}{lc} 
Material & Thickness (nm) \\
\hline $\mathrm{TiO}_{2}$ & 74 \\
$\mathrm{SiO}_{2}$ & 82 \\
$\mathrm{TiO}_{2}$ & 77 \\
$\mathrm{SiO}_{2}$ & 94 \\
$\mathrm{TiO}_{2}$ & 76 \\
$\mathrm{SiO}_{2}$ & 148 \\
$\mathrm{TiO}_{2}$ & 76 \\
$\mathrm{SiO}_{2}$ & 90 \\
$\mathrm{TiO}_{2}$ & 71 \\
$\mathrm{SiO}_{2}$ & 91 \\
$\mathrm{TiO}_{2}$ & 74 \\
\hline
\end{tabular}

performance (simulated on TFCalc ${ }^{\mathrm{TM}} 3.5$ ). Thus, the resonant cavity thickness was programmed to $160 \mathrm{~nm}$, instead of $206 \mathrm{~nm}$.

\subsection{Optical Filter Transmittance}

The transmittance of the fabricated optical filter was measured and shown in Fig. 5. The transmittance measurement was performed using the borosilicate glass substrate and an optical setup comprising: a quartz tungsten halogen lamp at $200 \mathrm{~W}$ (Newport 6334NS) with a light incident angle of 0 deg, a picoammeter (Keithley 487), a monochromator (Newport 74125), an optical fiber (Newport 77563), and a commercial photodiode (Hamamatsu S1336-5BQ). Moreover, it is also presented the simulated transmittance of the optical filter (on TFCalc ${ }^{\mathrm{TM}} 3.5$ ) using the thickness of each layer shown in Table 2 . The optical results are close to the ones obtained with the profilometry results (Table 2). The transmittance peak of the fabricated optical filter is

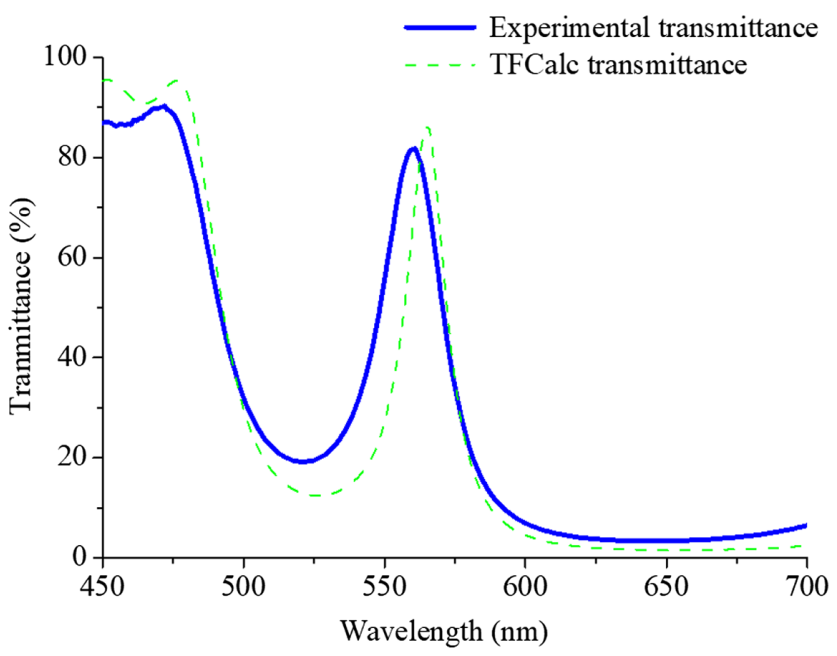

Fig. 5 Experimental transmittance of the fabricated optical filter and the transmittance obtained with the TFCalc ${ }^{\mathrm{TM}} 3.5$ using the experimental thicknesses of Table 2. $\sim 81.8 \%$ at $561 \mathrm{~nm}$, with an FWHM of $\sim 28 \mathrm{~nm}$. The experimental transmittance measurements and the simulations on TFCalc ${ }^{\mathrm{TM}}$ 3.5 were performed with 0 -deg light incident angle, which is important to ensure a correct comparison between the experimental and theoretical results. Moreover, 0-deg light incident angle is appropriated to the final device configuration, where the illumination is in the same face of the detection.

\subsection{Responsivity}

The responsivity of the CMOS silicon photodiode with the optical filter deposited on its top is shown in Fig. 6. The responsivity measurement was performed using a pinhole with $100-\mu \mathrm{m}$ diameter in one commercial photodiode (Hamamatsu S1336-5BQ), used as a reference to extract the CMOS photodiode with the optical filter responsivity curve. Moreover, the measurement was performed using an optical setup comprising: a quartz tungsten halogen lamp at $200 \mathrm{~W}$ (Newport 6334NS) with a light incident angle of $0 \mathrm{deg}$, a picoammeter (Keithley 487), a monochromator (Newport 74125), and an optical fiber (Newport 77563). The responsivity experimental measurement was performed with 0-deg light incident angle, which is appropriated to the final device configuration, where the illumination is in the same face of the detection. The responsivity presents a peak of $\sim 273.6 \mathrm{~mA} / \mathrm{W}$ at $578 \mathrm{~nm}$. As previously referred, the interface between the optical filter and the CMOS silicon photodiodes is a very thin oxide passivation layer $\left(\mathrm{SiO}_{2}\right.$ thin film with $\sim 20 \mathrm{~nm}$ ). This layer is crucial to the final performance of the CMOS photodiodes with the optical filter deposited on their top. The authors have previously tested the direct deposition of the optical filter on the photodiodes top without the passivation layer, which resulted in a poor performance of the set. Moreover, the CMOS silicon photodiode responsivity values shown in Fig. 6 are slightly increased when comparing with Fig. 2. This can be due to light alignment issues during the responsivity characterizations, using a pinhole with $100-\mu \mathrm{m}$ diameter in one commercial photodiode (Hamamatsu S1336$5 \mathrm{BQ}$ ), used as a reference to extract the responsivities curves (Figs. 2 and 6). Finally, the optical filter has a transmittance peak at $561 \mathrm{~nm}$ and the CMOS photodiode with the optical filter has a responsivity peak at $578 \mathrm{~nm}$. This difference can be related with two factors: the interface between the optical filter and the CMOS silicon photodiodes, an extra 20-nm-thick $\mathrm{SiO}_{2}$ layer;

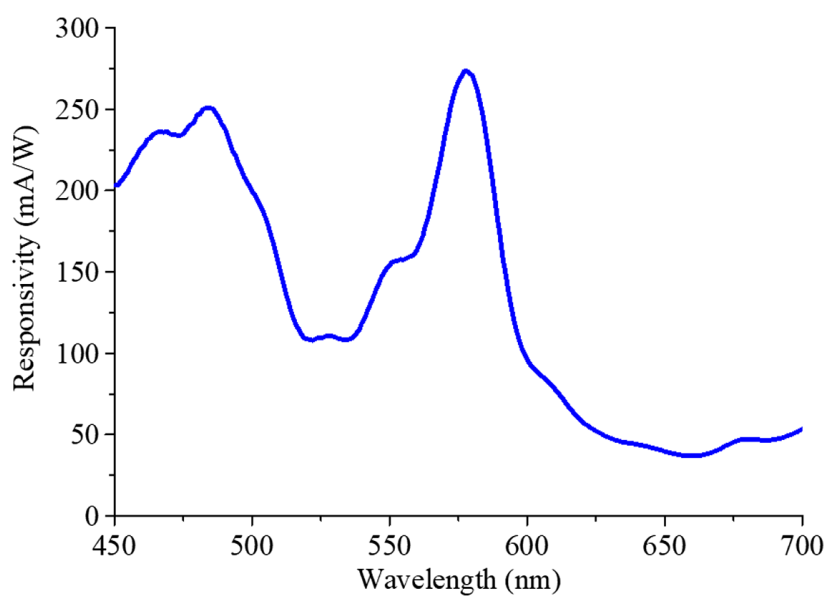

Fig. 6 Responsivity as a function of wavelength for the CMOS silicon photodiode with the optical filter deposited on its top. 


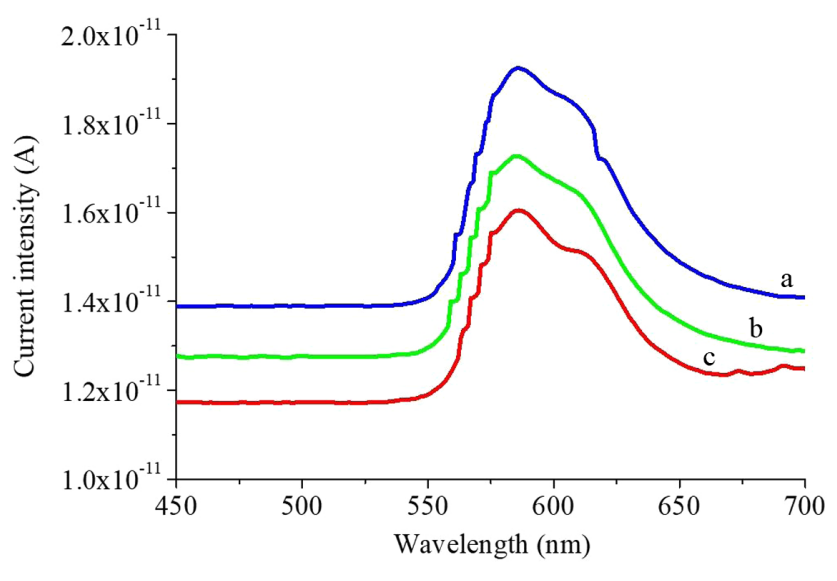

Fig. 7 Emission spectra of the fluorophore for several concentrations: curves a $(0.023 \mathrm{mg} / \mathrm{mL}), \mathrm{b}(0.017 \mathrm{mg} / \mathrm{mL})$, and c $(0.014 \mathrm{mg} / \mathrm{mL})$.

and the characteristic responsivity curve of the CMOS photodiode, small increase for higher wavelengths.

\section{Fluorescence Measurements: Results and Discussion}

\subsection{Fluorophore Characterization}

For the characterization of the implemented optical filter in the neural probe, it was used a fluorophore with excitation and emission properties similar to the O-GECO1. The molecule (C2734 Sigma-Aldrich, 5-Carboxytetramethylrhodamine) is specified with an excitation wavelength of $\sim 543 \mathrm{~nm}$ and an emission wavelength of $\sim 570 \mathrm{~nm}$, in methanol. ${ }^{17}$ To clarify these features, it is important to perform a spectral characterization of the molecule. Fluorescence measurements were performed using a commercial setup and considering an excitation light wavelength of $543 \mathrm{~nm}$. The commercial setup comprised: a quartz tungsten halogen lamp at $200 \mathrm{~W}$ (Newport 6334NS), a monochromator (Newport 74125) fixed at $543 \mathrm{~nm}$, a second monochromator (Oriel Instruments 74000) to measure the emission fluorescence, an optical fiber (Newport 77563), a picoammeter (Keithley 487), and a commercial photodiode (Hamamatsu S1336-5BQ).

The fluorescence measurements allowed the determination of the fluorophore emission spectrum as shown in Fig. 7. The curves a, b, and c correspond to different fluorophore concentrations, $0.023,0.017$, and $0.014 \mathrm{mg} / \mathrm{mL}$, respectively. The fluorophore emits fluorescence especially between 550 and $650 \mathrm{~nm}$, with an emission peak at $\sim 585 \mathrm{~nm}$. Thus, the peak responsivity of the CMOS silicon photodiode with the optical filter deposited on its top, implemented in the neural probe, covers the fluorophore emission (see Fig. 6).

\subsection{In-Vitro Characterization}

In-vitro fluorescence measurements were performed to validate the fabricated optical filter on the top of the CMOS silicon photodiodes array, implemented in the neural probe. It was used a front face geometry, which means illumination in the same face of the detection.

The obtained CMOS photodiode current as a function of the fluorophore concentration is shown in Fig. 8. The photodiode current is proportional to the fluorophore concentration. Moreover, the results show a good linearity as the $R^{2}$ value is

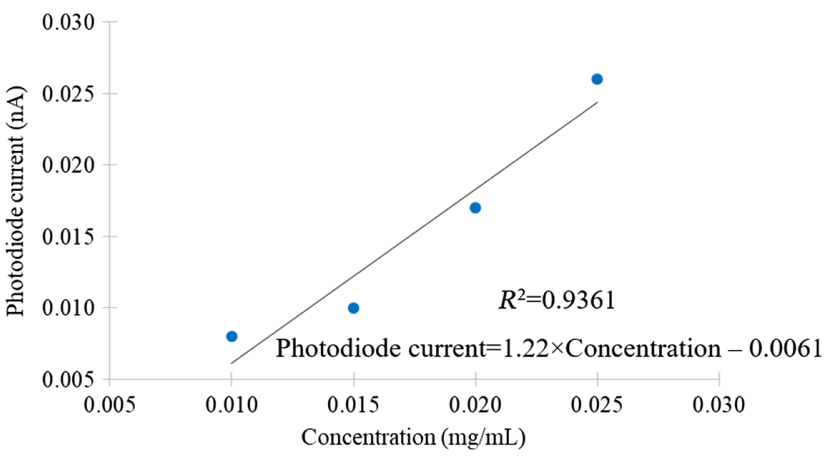

Fig. 8 CMOS silicon photodiode current as a function of the fluorophore concentration.

close to the unity, which validates the use of the neural probe with the high-selectivity optical readout function to determine the presence of different concentrations of a fluorophore, with excitation and emission properties similar to O-GECO1.

The neural probe integrating two commercial GaN/sapphire LEDs from Roithner LaserTechnik GmbH and the CMOS silicon photodiodes array with a selectivity Fabry-Perot optical filter deposited on its top is shown in Fig. 9. The commercial blue LED ensures $1 \mathrm{~mW} / \mathrm{mm}^{2}$ emission irradiance for optogenetic activation. ${ }^{18}$

Figure 10 shows a general performance of the developed device. The developed device rejects part of the fluorophore excitation light, which is important to measure the fluorescence emission with a low interference from the excitation light. However, the device does not reject light from photostimulation. This may not seem like an issue as the photostimulation can be performed only during a few seconds during the fluorescence acquisition, as reported by Kobayashi et al. ${ }^{9}$ Nevertheless, the control of the thickness of the thin films that forms the optical filter may be improved on the future, improving the calibration of the quartz crystal sensor used for real-time thickness measurement during each deposition. Improving the thin films thicknesses, the performance of the filter will also improve, concerning the rejection of photostimulation and excitation lights. Finally, the developed device presents a great advantage as the optical filter on the top of the CMOS photodiodes array is a multilayer of dielectric materials, which are materials with low absorption coefficient, being the optical filter transmittance complementary to its reflectance.

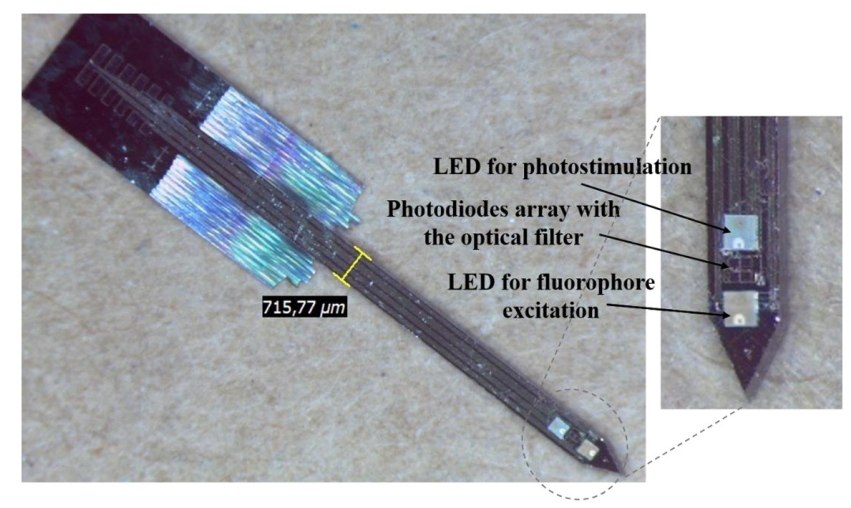

Fig. 9 Neural probe integrating the commercial LEDs and the CMOS silicon photodiodes array with the high-selectivity Fabry-Perot optical filter. 


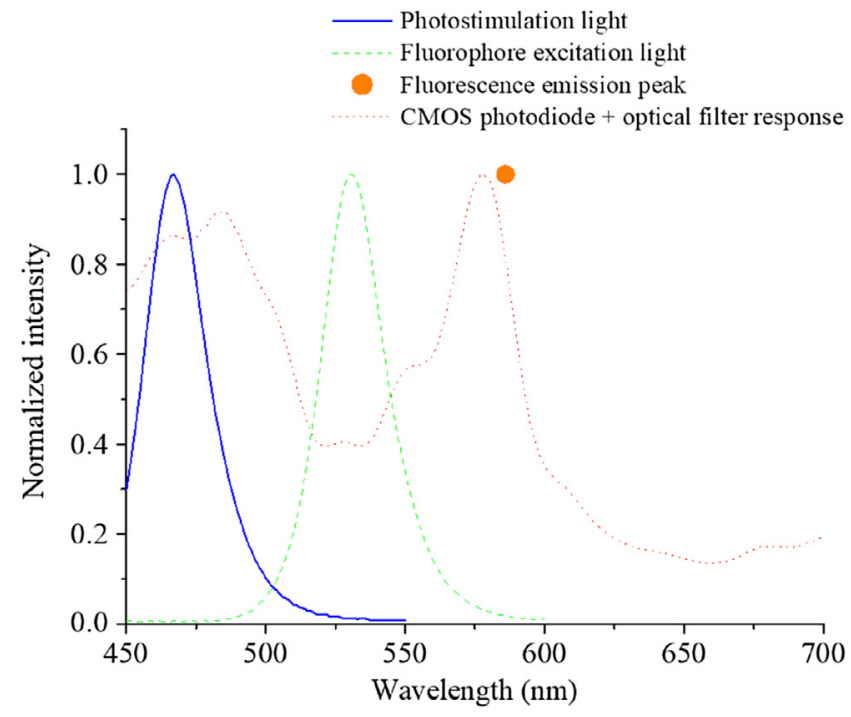

Fig. 10 General performance of the developed device.

\section{Conclusions}

This paper validates the use of a high-selectivity Fabry-Perot optical filter on the top of a CMOS silicon photodiodes array, implemented in a neural probe, for the detection of fluorescence signals, emitted by a fluorophore with excitation, and emission properties similar to O-GECO1.

The $\mathrm{SiO}_{2} / \mathrm{TiO}_{2}$ Fabry-Perot optical filter was fabricated by RF sputtering and has a transmittance of $\sim 81.8 \%$ at $561 \mathrm{~nm}$ and an FWHM of $\sim 28 \mathrm{~nm}$. The responsivity of the CMOS silicon photodiode with the optical filter deposited on its top is $\sim 273.6 \mathrm{~mA} / \mathrm{W}$ at $578 \mathrm{~nm}$. The interface between the optical filter and the CMOS silicon photodiodes array is a very thin layer of $\mathrm{SiO}_{2}$ (20-nm thick), crucial for a good performance of the set. In-vitro fluorescence measurements with a fluorophore showed a CMOS photodiode current proportional to the fluorophore concentration, with a good linearity $\left(R^{2}=0.9361\right)$.

The development of all device in a conventional CMOS process (only a postprocessing module to build the optical filter was added) allows on-chip electronics readout. The future implementation of a CMOS silicon photodiodes array, with higher density and lower photodiode size, in a neural probe can provide enhanced spatial resolution for future in-vivo fluorescence measurements. Additionally, an optical filters array can be implemented and the thickness of the resonator $\mathrm{SiO}_{2}$ layer, which is enclosed between the two dielectric mirrors, can be used as the gap-setting layer for obtaining four different optical filters, i.e., only controlling the thickness of the resonator $\mathrm{SiO}_{2}$ layer, an optical filter for a specific spectral range of interest can be obtained. This key advantage enables us to easily obtain miniaturized and low-cost narrow bandwidth multispectral devices. ${ }^{19}$

\section{Disclosures}

The authors have no relevant financial interests in the manuscript and no other potential conflicts of interest to disclose.

\section{Acknowledgments}

This work was supported by FCT with the reference project UID/EEA/04436/2013, by FEDER funds through the COMPETE 2020-Programa Operacional Competitividade e
Internacionalizacão (POCI) with the reference project POCI01-0145-FEDER-006941 and project PTDC/CTM-REF/28406/ 2017 (02/SAICT/2017), OpticalBrain. ANI also supports this work through the Brain-Lighting project by FEDER funds through Portugal 2020, COMPETE 2020 with the reference POCI-01-0247-FEDER-003416. S. B. Goncalves was supported by the Portuguese Foundation for Science and Technology, grant PD/BD/105931/2014, MIT-Portugal program.

\section{References}

1. W. H. Organization, "Mental disorders-fact sheet," http://www.who .int/mediacentre/factsheets/fs396/en/ (19 April 2018).

2. S. Myllymaa, K. Myllymaa, and R. Lappalainen, "Flexible implantable thin film neural electrodes," in Recent Advances in Biomedical Engineering, G. R. Naik, Ed., pp. 165-190, InTech Europe, Rijeka, Croatia (2009).

3. R. Khilwani et al., "Ultra-miniature ultra-compliant neural probes with dissolvable delivery needles: design, fabrication and characterization," Biomed. Microdevices 18, 1-20 (2016).

4. M. Azimipour et al., "Extraction of optical properties and prediction of light distribution in rat brain tissue," J. Biomed. Opt. 19, 075001 (2014).

5. S. B. Goncalves et al., "Design and manufacturing challenges of optogenetic neural interfaces: a review," J. Neural Eng. 14, 041001 (2017).

6. M. Choi et al., "Label-free optical activation of astrocyte in vivo," J. Biomed. Opt. 16, 075003 (2011).

7. M. Mesradi et al., "Experimental and analytical comparative study of optical coefficient of fresh and frozen rat tissues," J. Biomed. Opt. 18, 117010 (2013).

8. R. T. LaLumiere, "A new technique for controlling the brain: optogenetics and its potential for use in research and the clinic," Brain Stimul. 4, 1-6 (2011).

9. T. Kobayashi et al., "Optical communication with brain cells by means of an implanted duplex micro-device with optogenetics and $\mathrm{Ca}^{2+}$ fluoroimaging," Sci. Rep. 6, 21247 (2016).

10. J. Wu et al., "Improved orange and red $\mathrm{Ca}^{2+}$ indicators and photophysical considerations for optogenetic applications," ACS Chem. Neurosci. 4, 963-972 (2013).

11. M. Oheim et al., "New red-fluorescent calcium indicators for optogenetics, photoactivation and multi-color imaging," Biochim. Biophys. Acta 1843, 2284-2306 (2014).

12. H. Takehara et al., "Intravital fluorescence imaging of mouse brain using implantable semiconductor devices and epi-illumination of biological tissue," Biomed. Opt. Express 6, 1553-1564 (2015).

13. M. Haruta et al., "Intrinsic signal imaging of brain function using a small implantable CMOS imaging device," Jpn. J. Appl. Phys. 54, 04DL10 (2015).

14. DISCO, "Electroformed bond blades NBC-ZSeries," https://www.disco .co.jp/eg/products/catalog/pdf/nbc-z.pdf (20 April 2018).

15. S. Pimenta et al., "Design and fabrication of $\mathrm{SiO}_{2} / \mathrm{TiO}_{2}$ and $\mathrm{MgO} / \mathrm{TiO}_{2}$ based high selective optical filters for diffuse reflectance and fluorescence signals extraction," Biomed. Opt. Express 6, 3084-3098 (2015).

16. M. F. Silva et al., "NBI optical filters in minimally invasive medical devices," IEEE J. Sel. Top. Quantum Electron. 22, 6803307 (2016).

17. Sigma-Aldrich, "C2734 Sigma-Aldrich 5-carboxytetramethylrhodamine," https://www.sigmaaldrich.com/catalog/product/sial/c2734?lang= pt\&region=PT (24 April 2018).

18. R. Scharf et al., "Depth-specific optogenetic control in vivo with a scalable, high-density $\mu$ LED neural probe," Sci. Rep. 6, 28381 (2016).

19. L. Kong et al., "Single sensor that outputs narrowband multispectral images," J. Biomed. Opt. 15, 010502 (2010).

Sara Pimenta received her MSc degree in biomedical engineering (major in medical electronics) from the University of Minho, Portugal, in 2012. In 2017, she received her PhD also in biomedical engineering at University of Minho, Portugal. Currently, she is a postdoctoral researcher at CMEMS-UMinho. Her current research interests include development of microsystems for biomedical applications, optics, neuroscience, and microfabrication. 
José $\mathbf{P}$. Pereira is currently working toward his MSc degree in industrial electronics and computers engineering with the CMEMS-UMinho, Portugal. His research interests include optical communication with brain cells, optical filters simulation and fabrication, and microtechnologies.

Nuno M. Gomes graduated in materials engineering in 2002 from University of Minho, Portugal. Currently, he is a researcher at CMEMS-UMinho. His research interests include microfabrication for optics applications.

João F. Ribeiro graduated in industrial electronics engineering (integrated master) from University of Minho, Portugal, in 2010. In 2015, he received his $\mathrm{PhD}$ at the same institution. During his $\mathrm{PhD}$, he spent 10 months at LRCS, University of Picardie Jules Verne, France. Currently, he is a postdoctoral researcher at CMEMS and an invited professor of industrial electronics department from the University of Minho. His professional interests are micro/nanofabrication technologies and integrated microsystems for biomedical applications.

Manuel F. Silva graduated in informatics from the Polytechnic Institute of Cávado and Ave, Portugal, in 2008. In 2010, he obtained his MSc degree on micro/nanotechnologies from the University of Minho, Portugal. In 2015, he received his PhD in industrial electronics from the same institution. His past work focused on thermoelectric materials and fabrication of energy harvesting systems. Since 2011, he is involved in the research of optical microsystems and materials technology for medical devices.
Sandra B. Goncalves graduated in biomedical engineering (integrated masters) with the major in medical electronics, at the University of Minho, Portugal, in 2012. Currently, she is a PhD student at University of Minho, over the MIT-Portugal Program within the EDAM focus-area. During her PhD, she spent one year at MIT, in Boston, USA, working in the Synthetic Neurobiology Group. Her scientific interests are in the design and development of MEMS, neuroscience, and optoelectronics and microfabrication technologies.

Graca Minas graduated in industrial electronics engineering in 1994, received her MSc degree in 1998, and her $\mathrm{PhD}$ in 2004, all from University of Minho, Portugal. She is an associate professor, at the Department of Industrial Electronics, University of Minho, developing her research work in CMEMS, where she is involved in biomedical microdevices, on-chip integration of electronic circuits, and cointegration of optical filters on CMOS technique and sensors.

José H. Correia graduated in physical engineering from the University of Coimbra, Portugal, in 1990. In 1999, he received his $\mathrm{PhD}$ at the Laboratory for Electronic Instrumentation, Delft University of Technology, the Netherlands, working in the field of microsystems for optical spectral analysis. Currently, he is a full professor in the Department of Industrial Electronics, University of Minho, Portugal. He was the general-chairman of Eurosensors 2003 and MME 2007. Guimarães, Portugal. 